\title{
Draft \\ On the Determination of Ejecta Structure and Explosion 4/4/03 Asymmetry from the X-ray Knots of Cassiopeia A
}

\author{
J. Martin Laming ${ }^{1} \&$ Una Hwang ${ }^{2}$
}

\begin{abstract}
We present a detailed analysis of Chandra X-ray spectra from individual ejecta knots in the supernova remnant Cassiopeia A. The spectra are fitted to give the electron temperature $T_{e}$ and (single) ionization age $n_{e} t$. These quantities are compared with the predictions of self similar hydrodynamic models incorporating time dependent ionization and radiation losses, and Coulomb electron-ion equilibration behind the reverse shock, for a variety of different ejecta density profiles described by a uniform density core and a power law envelope. We find that the ejecta close to the "jet" region in the NE, but not actually in the jet itself, have a systematically shallower outer envelope than ejecta elsewhere in the remnant, and we interpret this as being due to more energy of the initial explosion being directed in this polar direction as opposed to equatorially. The degree of asymmetry we infer is at the low end of that generally modelled in asymmetric core-collapse simulations, and may be used to rule out highly asymmetric explosion models.
\end{abstract}

\section{Introduction}

It has become reasonably well accepted that the star recorded in the astronomer Flamsteed's 1725 catalogue as 3 Cassiopeiae was indeed the supernova that gave rise to the

${ }^{1}$ Code 7674L, Naval Research Laboratory, Washington DC 20375

jlaming@ssd5.nrl.navy.mil

${ }^{2}$ Goddard Space Flight Center and University of Maryland

hwang@orfeo.gsfc.nasa.gov 
remnant now known as Cassiopeia A (Ashworth 1980). Flamsteed's observation in 1680 makes Cas $A$ one of a handful of historical supernova remnants for which we have a precise age. In fact Cas $\mathrm{A}$ is by now well studied by astronomers at all wavelengths from the radio (it is the brightest source in the northern sky, Reber 1944; Ryle \& Smith 1948) to TeV $\gamma$-rays (Aharonian et al. 2001), allowing determinations with a high degree of confidence of important parameters such as the expansion velocities of various parts of the remnant (Fesen 2001) and its distance (Reed et al. 1995). This makes it an attractive "laboratory" for studying various physical phenomena, such as the plasma physics connected with particle acceleration (Vink \& Laming 2003), or nucleosynthesis through observed element abundances (see e.g. Vink et al. 2001).

In this paper we analyze the X-ray emission from small localized knots of material within the Cas A ejecta in order to try and constrain the ejecta density profile. The sub-arcsecond spatial resolution of the Chandra X-ray Observatory has made it possible to study individual structures in the Cas A shell. These structures have angular dimension typically $\sim 1^{\prime \prime}$, which is $\sim 5 \times 10^{16} \mathrm{~cm}$ at the $3.4 \mathrm{kpc}$ distance of Cas A. Consequently a reverse shock of velocity $1000 \mathrm{~km} \mathrm{~s}^{-1}$ (a typical value in our models below) will traverse these structures in around 20 years, a time significantly shorter than the evolution time of Cas A. Thus they may be fit with a single value of the ionization age $n_{e} t$, and models for the evolution of the SNR may be applied to infer the timing of the shock heating in a similar way to the use of nuclear reaction rates elsewhere in astrophysics, as a powerful tool for investigating the structure of Cas A. We emphasise that our goal is not to try and analyze the complete emission from the ejecta of Cas A, but that by isolating the X-ray knots we are focussing on those structures in Cas A for which we think we have the best physical understanding. In this paper we treat only those knots that appear to be O-rich, in order to constrain the ejecta density profile and possible asymmetries in the initial explosion. A companion paper considers in more detail a set of Fe rich knots on the east limb, determining element abundances for these knots, and from estimates of the Lagrangian mass coordinate for each knot, makes an initial attempt to build up a radial profile of the composition of the supernova ejecta.

\section{The Circumstellar Density Profile}

The forward shock speed in Cas A has been recently measured from the two extant Chandra ACIS observations with substantial exposures to be in the range $\sim 4000-6000$ $\mathrm{km} \mathrm{s}^{-1}$ (Delaney \& Rudnick 2003), with an average value of $4916 \mathrm{~km} \mathrm{~s}^{-1}$. This is consistent with previous estimates based on the observed expansion of the bright ejecta ring (Vink et al. 1998; Koralesky et al. 1998; Gotthelf et al. 2001). The average radius is 2.46 pc, leading 
to an expansion parameter of 0.654 . The fact that the ejecta are still clearly visible in X-rays has suggested to various authors that Cas A must be in transition from ejecta dominated to Sedov-Taylor behavior, and the expansion parameter of 0.654 appears most consistent with a uniform circumstellar medium, for which it should vary between $1 \rightarrow 0.4$ in these two limits. Simple interpretations of the spectra of forward and reverse shocked material also indicate that the masses of emitting ejecta and swept up circumstellar gas are similar (Fabian et al. 1980).

Based on optical spectra, however, the ejecta of Cas $\mathrm{A}$ are believed to be rich in $\mathrm{O}$ (Chevalier \& Kirshner 1979), and if this were the dominant constituent of the ejecta, the mass inferred from the thermal bremsstrahlung emission measure would be lower by approximately a factor of 4 than if it were of an $\mathrm{H}$ or He dominated composition. Vink, Kaastra, \& Bleeker (1996) were the first authors to make this point with regard to X-ray observations, and inferred an ejecta mass of $\sim 4 M_{\odot}$. Favata et al. (1997) revised this mass estimate to the range $2-4 M_{\odot}$ with further uncertainty arising from how the spectral component responsible for the hard X-ray emission behaves at lower (i.e. thermal) photon energies. Recently Willingale et al. (2003) also inferred an ejecta mass of $2.2 M_{\odot}$. These lower ejecta masses imply that the Cas A blast wave has swept up a relatively higher mass of circumstellar material, dominated in composition by $\mathrm{H}$ and $\mathrm{He}$, with $\mathrm{N}$ also over abundant relative to solar values. In this case we expect Cas A to have evolved to the Sedov-Taylor limit, and the observed expansion factor would imply a stellar wind solution for the circumstellar density profile, i.e. $\rho \propto r^{-2}$. The existence of a pre-supernova stellar wind is also suggested by the quasi-stationary flocculi (van den Bergh 1971), which are presumed to have formed from stellar wind material.

Another line of argument pointing to a stellar wind density. profile is from the existence of ejecta at optical emitting temperatures. The reverse shock encounters the densest ejecta at the core-envelope boundary, and these are the ejecta most likely to undergo thermal instability during the evolution of a young supernova remnant. In the case of a uniform circumstellar medium, for typical SNR parameters, the reverse shock only penetrates the ejecta core after a time of order 100 years, by which time the ejecta have expanded to such an extent that the radiative cooling time is now much longer than the remnant age. However with a stellar wind ambient medium, the initial deceleration of the blast wave sends the reverse shock into the ejecta core on a timescale of order a few years, when the ejecta are still dense enough to radiate significantly. This point was first made by Chevalier \& Fransson (1994), and is illustrated in Tables 1 and 2 where the mass of ejecta (assumed pure oxygen) that can cool to $10^{4} \mathrm{~K}$ within 320 years of explosion is given for various ejecta envelope power laws. Cas $\mathrm{A}$ is a little unusual in that it does indeed have optically emitting ejecta, both in knots and in nebulosity, again strongly suggesting an $s=2$ or similar ambient density profile. The existence of radiatively cooled shocked ejecta may also be relevant to the free-free 
absorption observed towards the center of Cas A in the radio (Kassim et al. 1995; Anderson \& Rudnick 1995). Explanations in terms of absorption by unshocked ejecta pose difficulties in that they require unphysically large masses of ejecta. Radiatively cooled shocked ejecta at temperatures of a few thousand $\mathrm{K}$ can be much more dense, hence dramatically reducing the mass of cool plasma required to give the necessary absorption, while producing negligible photoelectric absorption of X-rays. The secular decrease of the radio emission from Cas A (Reichart \& Stephens 2000) has also been interpreted in terms of the adiabatic expansion of electrons trapped in a shock wave where the magnetic field is also secularly decreasing, as one would expect in a remnant circumstellar wind (Berezhko, Pühlhofer, \& Völk 2003).

Weaker arguments in favor of an $s=2$ external density profile are discussed in Vink \& Laming (2003). The hard X-ray emission out to $\sim 100 \mathrm{keV}$ photon energy has been interpreted as due to non-thermal bremsstrahlung from a population of electrons accelerated by lower hybrid waves generated by secondary shocks within the Cas A shell (Laming 2001a,b). Bremsstrahlung is a relatively inefficient emission process; much more of the electron energy is dissipated as heat by Coulomb collisions between accelerated and ambient electrons. Relative to $s=0$, an SNR expanding into an $s=2$ circumstellar medium expands faster at later times, and this extra adiabatic expansion allows more heat to be deposited in the plasma without a large increase in temperature. Additionally, the existence of thermal instability in certain regions of the ejecta allows a much higher power loss by radiation. Consequently, in as far as one is prepared to accept the model of Laming (2001a,b), (and this is discussed at some length in Vink \& Laming 2003), a stellar wind external density profile is much more plausible than a uniform ambient density. In the same manner, non thermal bremsstrahlung emission models are much easier to construct in more radiative heavy element dominated ejecta than in an $\mathrm{H}$ or He dominated plasma. More recently Delaney \& Rudnick (2003) encountered difficulties in trying to interpret their measurements of the forward shock velocity and radius in terms of models using constant ambient density from Truelove \& McKee (1999). Setting the ratio of the forward and reverse shock radii at approximately 3:2 (Gotthelf et al. 2001), the models overpredict the observed free expansion rate (determined from outlying optical knots). The inferred ejecta mass is also rather small at $1.4 M_{\odot}$. Moving to the $s=2$ models described below (see section 3.3), the $n=9$ models give the correct ratio of the forward and reverse shock ratio, the correct relationship between the forward shock and free expansion rates, and a more plausible ejecta mass of $2 M_{\odot}$. Looking more closely at Figure 2 of Gotthelf et al. (2001), the ratio of forward to reverse shock ratio actually varies, being about 1.5 on the west limb, but larger $\sim 1.8-1.9$ on the east limb, suggesting a lower value for the ejecta envelope power law in this region. This observation will be interpreted in more quantitative detail below. 


\section{The Ejecta Density Profile}

\subsection{Are the Ejecta Knots really Knots?}

A significant fraction of the ejecta of Cas A are observed in X-rays to be in knots or clumps. Some of these are located very close to the forward shock position, inviting the somewhat naive assumption that they are significantly overdense compared to their surroundings. We argue below based on a number of lines of reasoning that these knots are not significantly overdense, but have high ionization ages due to encountering the reverse shock early in the evolution of Cas A. Their apparent positions close to the blast wave may possibly be due to efficient particle acceleration and stronger resulting shock compression at the forward shock, as modelled by Blondin \& Ellison (2001). However we find no other indications in Cas A, either morphological or spectral, of shock compressions greater than 4 that would then be expected, and we do not pursue this issue further in this paper.

If these knots did indeed undergo reverse shock passage early in the evolution of Cas A, then an important question arises as to how they survived to be observed as knots by Chandra/ACIS. A number of authors (Wang \& Chevalier 2001; Klein, McKee \& Colella 1994; Klein et al. 2003; McKee \& Cowie 1975; Poludnenko, Frank, \& Blackman 2001) have modelled cloud-shock or knot-shock interactions. Upon entering a higher (lower) density medium, the shock decelerates (accelerates). The cloud or knot undergoes a different acceleration upon shock passage relative to the ambient plasma, which can give rise to a Kelvin-Helmholtz instability at the interface. In addition the shock inside the cloud or knot will be split into transmitted and reflected shocks each time it encounters the cloud/knot boundary with the ambient plasma, giving rise to further Rayleigh-Taylor or Richtmeyer-Meshkov instabilities. The net result is the destruction of the cloud or knot on a timescale of a few shock crossing times. For knots $1^{\prime \prime}$ across in Cas A $\left(5 \times 10^{16} \mathrm{~cm}\right)$ the shock crossing time is $15-30$ years for a $500-1000 \mathrm{~km} \mathrm{~s}^{-1}$ reverse shock. The knot destruction time of $\sim 50$ years is consistent with observed lifetimes of the optical fast moving knots and quasi-stationary flocculi (Thorstensen, Fesen, \& van den Bergh 2001; Sutherland \& Dopita 1995; van den Bergh \& Kamper 1985; Kamper \& van den Bergh 1976). However the X-ray knots of interest here are of similar temperature to their surroundings (i.e., they have not cooled to optically emitting temperatures), and their apparent survival for $\sim 200-300$ years (for models in Table 1) places an upper limit on their density with respect to the surrounding plasma of around a factor of 3 (see Table 3 of Klein, McKee \& Colella 1994). These authors did not consider underdense knots, but the simulations of Blondin, Borkowski \& Reynolds (2001) suggest that these would be no less unstable. The X-ray knots do not appear to coincide with the fast moving optical knots (FMKs), which do appear to be significantly overdense and are currently undergoing shock interaction. We believe the X-ray knots underwent reverse shock 
passage, and perhaps interacted with secondary shocks, some time early in the evolution of Cas $\mathrm{A}$ and are now expanding with the rest of the remnant plasma, as in fact appears to be the case (Delaney \& Rudnick 2003).

$\mathrm{X}$-ray knots of similar density to the surroundings are also much more consistent with the apparent factor of only 3-4 increase in surface brightness compared to the ambient plasma. Consider a knot with solar abundance ratios of $\mathrm{Si}, \mathrm{S}, \mathrm{Ar}, \mathrm{Ca}$, etc relative to $\mathrm{O}$ compared to a pure $\mathrm{O}$ knot. At temperatures around $1 \mathrm{keV}$ the $\mathrm{Si}-\mathrm{Ca}$ is predominantly in $\mathrm{He}$ - and H-like charge states and emits about 30 times more power per ion $(\sim 15$ times more power per unit mass; Summers \& McWhirter 1979) than O, which is fully ionized and radiates only by thermal bremsstrahlung. A knot composed of $0.75 \mathrm{O}$ and $0.25 \mathrm{Si}$-Ca by mass will thus radiate nearly 5 times more energy than a pure $O$ knot integrated over photon energy. When considering just emission in the X-ray bandpass, the knot with heavy elements will radiate even more compared with the pure $O$ knot by an extra factor $\sim \exp \left(E / k_{\mathrm{B}} T_{e}\right) \simeq 2$, where $E$ is the lower limit of the bandpass. This occurs because most of the $O$ thermal bremsstrahlung power is radiated outside the $\mathrm{X}$-ray band of interest, but the line emission from the heavier elements is not. A knot of dimension $\sim 10^{17} \mathrm{~cm}$ compared with background plasma extending over $1 \mathrm{pc}$ in depth needs to be around 30 times more radiative in order to be visible as a distinct object, so a density enhancement of around 2, giving 4 times more radiation, is all that is required. The knots fitted in Table 1 all have Si/O abundance ratios typically $0.5-1$ times the solar value (relative to the most recent value of the solar O abundance, Allende Prieto, Lambert, \& Asplund 2001; Holweger 2001), and also usually have small amounts of Fe which we have not considered here. The knot visibility for minimal density enhancement becomes even stronger if the surrounding plasma is in part $\mathrm{H} / \mathrm{He} / \mathrm{N}$ dominated composition, as would be expected for circumstellar medium shocked by the blast wave or ejecta shocked very early by the reverse shock.

\subsection{Observations and Data Analysis}

For our spectral analysis of ejecta knots in Cas A, we use the $50 \mathrm{ksec}$ observation with the Chandra Advanced CCD Imaging Spectrometer from January 2000. The observation uses the backside-illuminated CCD S3 in GRADED mode, wherein each CCD event is classified by its distribution over the detector pixels before telemetry from the spacecraft. We have considered three radial series of knots to the east, northeast at the base of the ejecta jet, and north-northwest, as shown in Figure 1. The knots have angular diameters of a few to several arcseconds, and are numbered starting closest to the center and progressing radially outward, except for the base of the ejecta jet, which is shown in more detail in Figure 2. For 
each knot, we typically extract a spectrum with several thousand counts prior to background subtraction.

The spectra are accumulated in pulse-invariant channels after correction for the spatial variation of the gain across the detector, but because the data were obtained in GRADED mode, the full removal of the effects of charge transfer inefficiency was not possible. Appropriate spatially weighted detector response functions and effective area functions were constructed for each spectrum. The background was taken from several regions off the source. For knots which generally have high surface brightness relative to the background (i.e., most of the knots in this sample), the spectral fits are not highly sensitive to the background subtraction. These data were obtained relatively early in the Chandra mission (198 days after launch) so the effect of the buildup of contaminants on the detector is relatively small, but we include a component in the spectral model to account for this excess absorption (ACISABS in XSPEC).

For each knot spectrum, we have fitted simple models for a single temperature plasma with a single ionization age. The spectral models used do not include emission from the element Ar, so a narrow Gaussian line is included in the model to represent the Ar He $\alpha$ blend near $3.1 \mathrm{keV}$. In all cases, the extremely strong emission lines indicate that the emission is dominated by the ejecta, but an additional continuum component is also required (as first noted by Hughes et al. 2000).

We have considered two sets of assumptions for the source of this continuum: the elements $\mathrm{H}, \mathrm{He}, \mathrm{C}$, and $\mathrm{N}$ in their solar abundance ratios, and an O-rich ejecta plasma in which $\mathrm{H}, \mathrm{He}, \mathrm{C}$, and $\mathrm{N}$, and $\mathrm{Ne}$ are absent and $\mathrm{O}$ provides the continuum. We favor the $\mathrm{O}$ continuum fits as they are more straightforwardly compared to the model calculations described above and are a more plausible description of the composition of the ejecta (after Vink et al. 1996). Both sets of fits give reasonably comparable fits, although in specific cases, either might at times give a better value fot the fit statistic than the other. We use the $\mathrm{O}$ continuum fit results, noting that the values of the fitted temperature and ionization age are typically affected by less than $15 \%$ and $40 \%$ respectively if the solar composition continuum is used instead, though the effect is larger in a few cases. A comparison of the fits for knots 4 in the E and NNW are shown in Figure 3 and Figure 4, for both O-rich and solar compositions.

Because of residual uncertainties in the gain of the detector (e.g., from charge transfer inefficiency), combined with the known spatially dependent radial velocities of the X-ray emitting gas (e.g., Markert et al. 1983), we have allowed the redshift of the model to be freely fitted; likewise a Gaussian smoothing function whose width scales with energy was also included in the model. 
Table 1 shows the results of the spectral fits for all three radial series, giving the temperatures, ionization ages, and abundances of the elements $\mathrm{Si}$ and $\mathrm{Fe}$ (by number relative to $\mathrm{O})$. In most cases, we obtain reasonable values of the fit statistic $\left(\chi^{2} /\right.$ dof $\left.\sim 1.5\right)$. Poorer fits (e.g., in the NNW and for the outer knots in the E) generally arise from the presence of Fe $\mathrm{K}$ emission in the spectrum that is not well-fitted by the model. The fits are driven strongly by the prominent $\mathrm{Si}$ line emission, but knots that are very rich in Fe relative to $\mathrm{Si}$ are described by different parameters than the knots with strong Si emission, particularly in having a higher ionization age. For knots that show a mixture of these characteristics, fully successful models would have to include components to describe $\mathrm{Si}$ and Fe separately.

In principle, the blast wave also contributes to each of the knot spectra. To verify that it is valid to neglect the blast wave contribution in examining these compact regions, we have compared the fit result for the well-isolated knot NNW1 using the standard offsource background compared to a local background region surrounding the knot. The local background should incorporate the blast wave contribution so that using this background spectrum effectively subtracts the blast wave contribution at the knot. We found no significant difference in the temperature $\left(\mathrm{kT}=0.93_{-0.09}^{+0.08} \mathrm{keV}\right.$ compared to $\mathrm{kT}=1.07_{-0.09}^{+0.12} \mathrm{keV}$ with the off source background), and a poorly constrained ionization age that is consistent with the value previously obtained.

\subsection{Ejecta Profile Models}

Our methods for computing the ejecta model follow from calculations in Laming (2001b), Laming \& Grun (2002) and Laming \& Grun (2003), and are summarized in the two appendices to this paper. We take an analytic approximation to the hydrodynamics for supernova ejecta expanding into a remnant stellar wind from Truelove \& McKee (1999), specified more thoroughly in Appendix A. Within this framework we calculation the time dependent ionization balance for the elemental composition of the ejecta, the electron and ion temperatures allowing only for equilibration between species by Coulomb collisions, and radiative and adiabatic expansion power losses, as outlined in Appendix B.

We have calculated two series of ejecta models, summarized in Tables 2 and 3 . Figures 5 and 6 show the locus of electron temperature, $T_{e}$, against ionization age, $n_{e} t$, for these sets of models. We concentrate on these parameters since they are readily determined from fits to the data, and the curves of $T_{e}$ against $n_{e} t$ are, for reasons discussed further below, surprisingly robust against various changes to the model, being mainly dependent on the ejecta density profile specified through the envelope power law $n$. Both sets of models have an ejecta mass of $M_{e j}=2 M_{\odot}$. The first has $E_{51}=3 \times 10^{51} \mathrm{ergs}$ explosion energy and an ambient density at 
the current blast wave position in the range 3.1-3.6 $\mathrm{H}$ atoms $\mathrm{cm}^{-3}$, depending on the value taken for the blast wave radius (i.e. $\rho r_{b}^{2}=21 \mathrm{H}$ atoms $\mathrm{cm}^{-3} \mathrm{pc}^{2}$ ). These values are chosen to match the blast wave velocity and radius measurements of Delaney \& Rudnick (2003) as closely as possible for values of $n \simeq 9$ (in the middle of our range). The second set has the same ejecta mass but an explosion energy of $2 \times 10^{51} \mathrm{ergs}$ and circumstellar density reduced from before by the same factor $2 / 3$. These models may be scaled keeping $E_{51} \propto M_{e j} \propto \rho r_{b}^{2}$ which leaves the blast wave velocity and radius invariant. The first set of models is chosen to match the blast wave velocity and radius found by Delaney \& Rudnick (2003), but implies a mass of shocked circumstellar material of close to $17 M_{\odot}$, significantly larger than the $\sim 9 M_{\odot}$ found by Willingale et al. (2003). The emission measure determined from thermal bremsstrahlung using the BeppoSAX data studied in Laming $(2001 \mathrm{a}, \mathrm{b})$ interpreted as coming from an H-He dominated plasma is consistent with masses of shocked circumstellar plasma in the range $6.7-10 M_{\odot}$ for densities ahead of the blast at its current location of $3-2 \mathrm{H}$ atoms $\mathrm{cm}^{-3}$ (or equivalent mass) respectively. These densities combined with the blast wave radius give masses of $11-16.8 M_{\odot}$ from simple geometrical considerations for densities of $2-3 \mathrm{H}$ atoms $\mathrm{cm}^{-3}$, and so it is clear that more consistent results derive from the lower circumstellar density. Using this however gives a radius for the blast wave that is slightly too small. We suspect the Cas A may have undergone an initial period of expansion into a tenuous WR wind before hitting the much denser red supergiant wind into which it is now expanding. We discuss this further in section 3.4.

In Tables 2 and 3 we give the predicted blast wave velocity, radius and expansion parameter $\eta$, the reverse shock radius and the ratio of the shock radii. This ratio is measured to vary between 1.52 and 1.73 with lower values being found in the west limb of Cas A (Gotthelf et al. 2001). From the models we can see that this immediately implies $n \simeq 9$ on the west limb decreasing to 6-8 elsewhere. The following table entries give dynamical parameters from the shock solutions, and the final column gives the mass of gas that may cool to optical emitting temperatures $\left(\sim 10^{4} \mathrm{~K}\right)$ within 320 years of explosion. This mass increases with $n$, being zero for the $n<7$ models. The initial inference of highest $n$ on the west limb is appealing since this is where the strongest radio and non-thermal X-ray emission is located coincident with the position of the contact discontinuity, (Bleeker et al. 2001; Gotthelf et al. 2001) implying that this is the location of strongest magnetic field. The high mass of radiatively cooled and thus very dense gas in this region will lead to stronger Rayleigh-Taylor instability at the contact discontinuity here than elsewhere, and hence a higher magnetic field. In what follows we will develop the idea of varying ejecta envelope power law with position in the remnant and try to quantify the degree of anisotropy by reference to our ejecta models.

We argued above that only a modest degree of overdensity may exist in the X-ray 
knots, in order for them to survive against hydrodynamic instabilities following reverse shock passage. Here we demonstrate that the clumping of ejecta by a factor up to around 3 does not greatly affect the dependence of $T_{e}$ on $n_{e} t$. Consider ejecta with a reference value of $n_{e} t$ at the current epoch, which encountered the reverse shock at time $t_{0}$. If this were overdense by a factor $\alpha$, say, then it would have passed through the reverse shock at a time $t_{c}=\alpha t_{0}$, since the electron density varies as $n_{e} \propto 1 / t^{2}$ and $\int_{t_{0}}^{t^{\prime}} n_{e} d t \propto 1 / t_{0}$ for $t_{0}<<t^{\prime}$. The clumped ejecta are shocked to a temperature lower by $1 / \alpha$ than the unclumped ejecta, but undergoes less cooling by adiabatic expansion. In ballistic expansion temperatures are reduced by adiabatic expansion by a factor

$$
T^{\prime}\left(t^{\prime}\right) \simeq T\left(t_{0}\right) \exp \left(-\frac{4}{3} \int_{t_{0}}^{t^{\prime}} \frac{v}{r} d t\right) \simeq T\left(t_{0}\right) \exp \left(-\frac{4}{3} \int_{t_{0}}^{t^{\prime}} \frac{d t}{t}\right) \simeq T\left(t_{0}\right)\left(\frac{t_{0}}{t^{\prime}}\right)^{4 / 3} .
$$

Hence clumped ejecta shocked at $\alpha t_{0}$ will have a final temperature higher than that for unclumped ejecta by a factor $\alpha^{-1} \times \alpha^{4 / 3}=\alpha^{1 / 3}$. In Sedov-Taylor expansion $r \propto t^{2 / 3}$ the final exponent $4 / 3$ become $8 / 9$, and clumped ejecta has a final temperature lower than that for unclumped ejecta by a factor $\alpha^{-1 / 9}$. Additionally, if $T_{i} \gg T_{e}$ so that $T_{e} \propto\left(T_{i} n_{e} t\right)^{2 / 5}$, (Laming 2001a), the temperature difference factor varies as $\alpha^{2 / 15} \rightarrow \alpha^{-2 / 45}$, remembering that the fits are sensitive only to $T_{e}$. Consequently only for large overdensities $\alpha \mathrm{m}$ will significant changes in $T_{e}$ result. We have verified with numerical calculations that these conclusions hold, using formulae given by Sgro (1975) for transmitted and reflected shock velocities.

It has been argued previously (Laming 2001a,b) that the non-thermal hard X-ray continuum of Cas A extending out to $100 \mathrm{keV}$ is bremsstrahlung from a population of electrons . accelerated by secondary shocks propagating within the shell. These shocks arise as the forward or reverse shocks encounter density contrasts and split into transmitted and reflected shocks. How justified are we in assuming that the ejecta knots of interest encounter the reverse shock and then expand self similarly with the rest of the plasma, undergoing no further interaction with secondary shocks? Following the discussion and analysis in Vink \& Laming (2003), we believe that the electron acceleration actually occurs in shocked circumstellar plasma in the immediate vicinity of the contact discontinuity, since an instability operating in colder shocked ejecta is much less likely to yield the required electron energies. Thus it is the blast wave encountering quasi-stationary flocculi that gives rise to the secondary shocks of interest, not the reverse shock. The ejecta knots we study here do in fact have Lagrangian mass coordinates that place them well back from the contact discontinuity, i.e. there is a lot of mass through which a secondary shock originating at the blast wave would have to propagate through to reach them, and we consider the possibility of significant heating by secondary shocks unlikely. The reverse shock is currently propagating through even more 
tenuous plasma than the forward shock, and again is unlikely to produce secondary shocks that perturb the ejecta knots. In our view, the ejecta were subject to various hydrodynamic instabilities following reverse shock passage and interactions with secondary shocks for a time comparable to the time taken for the reverse shock to propagate further into lower density ejecta. What we now see as "knots" are those structures that were able to survive against these instabilities, due to having little density contrast with their surroundings.

\subsection{Discussion}

Figures 5 and 6 plot the locus of $T_{e}$ against $n_{e} t$ for the two sets of ejecta models, with results from those spectral fits for which the $\chi^{2}$ per degree of freedom is less than 2 to the knots superimposed. In both cases the knots from the jet base on the NE limb consistently

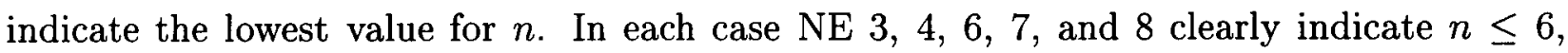
and NE 5 (not plotted) is also consistent with this. NE 9 and 10 indicate steeper envelopes, $n=7-9$ depending on the choice of model and NE 1,2, and 11 suggest $n \geq 12$ or $n=6$ if coming from the envelope portion of the curve. We suspect that this relatively large change in $n$, if real, over small apparent distances is mainly due to projection effects, knots NE 1,2

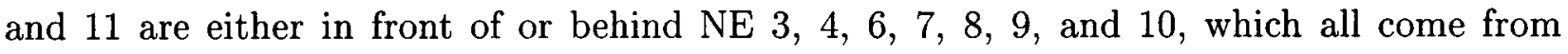
the brightest part in this region. Comparing to Delaney \& Rudnick (2003), our knots are closest to their regions 2-5 from which they measured forward shock velocities in the range $4361-6520 \mathrm{~km} \mathrm{~s}^{-1}$ and radii $2.08-2.43 \mathrm{pc}$. The series of knots on the east limb all indicate $n \simeq 9$ or higher, except for knots E1 and E2 which are the two nearest the projected SNR center and are closer to $n=6$. Delaney \& Rudnick (2003) do not take any measurements from this region of the blast wave, the closest being their region 29 some distance to the south. The NNW series of knots pose more problems in fitting the Fe $\mathrm{K}$ region, but those for which adequate fits can be found, NNW 4 indicates $n=6$ and NNW 5 and 7 give $n=7-12$, depending on the hydrodynamic model, with $n=7-8$ being preferred. These knots are closest to regions 13 and 14 in Delaney \& Rudnick (2003). At this point we give more attention to the model with $E_{51}=2$ and $\rho r_{b}^{2}=14$. Reasons for doing so are given above with reference to the shocked circumstellar material and the geometry of the blast wave. We also find that the lowest temperature knots for the higher energy model are only consistent with ejecta envelope power laws $n=30-50$ which are much steeper than any realistic explosion model would predict. Hydrogen rich ejecta give electron temperatures lower for a given $n_{e} t$ by a factor 2-3. More electrons per baryon means that the equilibrated electron temperature will always be a factor of 2 lower than for O. Additionally, the lower ion charge leads to slower electron-ion equilibration before this point is reached. We consider that these points reinforce our assumption of O-rich ejecta. 
We would like to interpret the different ejecta envelope power laws as due to asymmetries in the explosion. The models of Tables 2 and 3 give different ejecta core densities. Varying $E_{51}$ to keep the core density invariant yields

$$
E_{51} \propto M_{e j}^{5 / 3} \frac{(n-3)^{5 / 3}}{n^{2 / 3}(n-5)} .
$$

and we can see that the lower values of $n$ actually correspond to higher explosion energy in that particular direction. An $n=6$ model corresponds to $66 \%$ more energy than $n=9$. The variation still leads to blast wave radii predicted to vary between 2.35 and $2.62 \mathrm{pc}$, with lower $n$ giving larger radii. This is similar to the range of blast wave radii found by Delaney \& Rudnick (2003), but is not completely consistent with the locations they measure, and so for the time being we further adjust all models to give the same blast wave radius. These new models are given in Tables 4 and 5 . The degree of explosion asymmetry one would infer from the shallower ejecta profile is now slightly reduced; $n=6$ corresponds to about $40 \%$ more energy than $n=9$ for our preferred hydrodynamic model. The curves of $T_{e}$ against $n_{e} t$ for these new models for $n=5.5$ and 6 are plotted as dotted lines on Figures 5 and 6 . For higher $n$ the new curves are negligibly different to the previous ones. We can see that for both hydrodynamic models the NE knots at the jet base now clearly suggest $n=6$ and not $n=5.5$, and other conclusions remain the same.

The degree of energy asymmetry in the initial explosion is at the lower end of that coming from simulations. Two basic mechanisms of asymmetric core collapse explosions have been discussed in the literature. Fryer \& Heger (2000) and Fryer \& Warren (2003) model the explosion of a rotating star in two and three dimensions respectively. Compared to nonrotating explosions, the rotating core appears to be stabilized against convection, and the core bounce that seeds the neutrino-driven convection is also weakened. Both these effects are reduced in the polar regions, and consequently an asymmetric explosion which is stronger in the polar regions results. Ejecta velocities about a factor of two higher in polar relative to equatorial regions are predicted, corresponding to a factor of four difference in kinetic energy if the density is the same in polar and equatorial regions. ${ }^{56} \mathrm{Fe}$ is generally synthesized (as ${ }^{56} \mathrm{Ni}$ ) along the axis. Additionally, the generic problem of core-collapse simulation producing too much neutron-rich matter appears to be slightly exacerbated, though Fryer \& Warren (2002) caution that much of the input physics upon which this conclusion depends is still uncertain. In contrast Khokhlov et al. (1999) model a jet induced explosion. This mechanism is independent of details of convection. A magnetorotational instability in the collapsing core (recently studied in more detail by Akiyama et al. 2003) accelerates two jets along the rotation axis. These drive bowshocks ahead of them that compress most of the remaining ejecta into an equatorial torus. Similar anisotropies in ejecta velocities to the rotating case above are found, though due to the anisotropic ejecta density distribution the kinetic energies 
will be less anisotropic.

Nagataki et al. (1998) postulate similar degrees of asymmetry in their models to explain high yields of ${ }^{44} \mathrm{Ti}$ produced in $\alpha$-rich freeze out in the polar regions. Their model A1, with similar asymmetry to models discussed above, produces $1.8 \times 10^{-4} M_{\odot}$ of ${ }^{44} \mathrm{Ti}$ and $0.06 M_{\odot}$ of ${ }^{56} \mathrm{Ni}$ for a suitable choice of mass cut. The ${ }^{44} \mathrm{Ti}$ mass is similar to that inferred for Cas A (Vink et al. 2001; Vink \& Laming 2003). The mass of ${ }^{56} \mathrm{Ni}$ produced is less reliably estimated from observations. In a companion paper (Hwang \& Laming 2003) we do indeed find evidence of ${ }^{44} \mathrm{Ti}$ production in $\alpha$-rich freeze out, not in the jet region but further out along the east limb series of knots. Indeed this, the general distribution of heavy elements in a torus around the "jet" axis, and the existence of the "jet" itself in Cas A do resemble the model discussed by Khokhlov et al. (1999), though considerably more detailed analysis would be required to confirm anything more quantitative.

The progenitor mass may now be estimated from the parameters of our models. Most of the stellar wind is expelled during the red supergiant phase, and it is into this wind that we believe Cas $\mathrm{A}$ is now expanding. A massive star spends $\sim 2 \times 10^{5}$ years in this stage (Garcia-Segura, Langer, \& Mac Low 1996), roughly independent of stellar mass. The mass loss rate in this phase is given by

$$
\frac{d M}{d t}=4 \pi \rho r^{2} v_{w}=3 \times 10^{-5}\left(\frac{\rho r_{b}^{2}}{1 \mathrm{H} \text { atom } \mathrm{cm}^{-3} \mathrm{pc}^{2}}\right)\left(\frac{v_{w}}{100 \mathrm{~km} \mathrm{~s}^{-1}}\right) M_{\odot} \text { year }^{-1},
$$

where $v_{w}$ is the stellar wind speed and our favored value for $\rho r_{b}^{2}=14$. We estimate $v_{w}$ from the speeds of the quasi-stationary flocculi of Cas A. In general, they are observed to have proper motions ranging up to around $500 \mathrm{~km} \mathrm{~s}^{-1}$ (van den Bergh \& Kamper 1985), consistent with clumps a factor of $\sim 10^{2}-10^{3}$ more dense than the ambient plasma having undergone some acceleration during the passage of the blast wave. The lowest observed velocity is $20 \mathrm{~km} \mathrm{~s}^{-1}$ for QSF 10 . Taking this value in equation (3) we estimate a total mass loss during the red supergiant phase of $17 M_{\odot}$. The iterated blast wave speeds and radii in Tables 4 and 5 are respectively slightly higher and lower than the average values in Delaney \& Rudnick (2003), with our second set of model for lower $\rho r_{b}^{2}$ being more discrepant. This might suggest an early stage of expansion into a much more tenuous stellar wind from a Wolf-Rayet progenitor, before the blast wave encounters the much more dense red supergiant wind, similar to a model for Cas A proposed by Borkowski et al. (1996). In the case of Cas A such a fast tenuous wind can not have existed for very long before explosion because one would then lose the radiative instability of shocked ejecta and the accompanying optical emission, and so the mass lost during this period is probably negligible. To the estimate above we should then add the observed mass of ejecta and compact objects of about 3-4 $M_{\odot}$ and mass loss during the main sequence evolution to get a total progenitor mass in 
the range $20-25 M_{\odot}$, at the lower end of Wolf-Rayet progenitor masses inferred by Massey, DeGioia-Eastwood, \& Waterhouse (2001). The assumed value for the wind speed of $20 \mathrm{~km}$ $\mathrm{s}^{-1}$ is significantly lower than is usually assumed for red supergiants (e.g. Garcia-Segura, Langer, \& Mac Low 1996; Lamers \& Nugis 2002) where speeds around $100 \mathrm{~km} \mathrm{~s}^{-1}$ seem more plausible. This would increase our mass estimate to close to $100 M_{\odot}$. This seems to us unlikely since the progenitor would then spend much longer in the Wolf-Rayet phase before explosion (Woosley, Langer, \& Weaver 1993), so much so that the Cas A blast wave should still be moving through Wolf-Rayet wind rather than red supergiant wind, and as discussed above, no thermal instability should be present in the ejecta. The Wolf-Rayet phase is much shorter for less massive stars, again arguing for a progenitor at the lower end of observed progenitor masses for Wolf-Rayet stars. These masses are consistent with those estimated from the initial ${ }^{44} \mathrm{Ti}$ mass inferred from $\gamma$ ray observations (Iyudin et al. 1994, 1997; Vink et al. 2001; Vink \& Laming 2003) compared with explosion calculations (e.g. Timmes et al. 1996). Rothschild \& Lingenfelter (2003) provide a more complete discussion of this point.

\section{Conclusions}

This paper has presented an initial analysis of the X-ray ejecta knot spectra from Cassiopeia A. Although considerably more labor intensive and detailed than any previous analysis of imaging spectroscopy of supernova remnants known to us, we believe that we are close to being able to infer some of the really fundamental aspects of this object. Rather than attempting to analyze and model the entire spectrum of the remnant, we have isolated spectra from ejecta knots whose properties we think we understand, which when combined with 1-D hydrodynamical models allow us to infer ejecta envelope density profile power laws and hence explosion energies in different directions in the remnant. The fundamental discriminant in this work is the variation of the reverse shock velocity in the models with different ejecta density profiles. The faster reverse shock in shallower profiles leads to higher ejecta temperatures and a larger separation between the forward and reverse shocks. These considerations lead to conclusions concerning the energy asymmetry of the explosion, which we find to be around $50 \%$ larger in polar regions than at equatorial regions outside the so-called jet. This is at the lower range of energy asymmetries coming from existing core-collapse explosion simulations, and on this basis alone we are not yet able to distinguish between rotating convection driven explosions or the jet induced explosions. Aside from the demonstration of new data analysis and interpretation techniques, the one further important conclusion we wish to emphasize is that only with significantly deeper Chandra observations of Cassiopeia A and other supernova remnants will the full potential of these methods be realized. 
We wish to thank Larry Rudnick and Tracey Delaney for communication of their results prior to publication, and particularly to Tracey Delaney for a careful check of some of the material in Appendix A. JML was supported by basic research funds of the Office of Naval Research.

\section{A. Hydrodynamics for $s=2$ Supernova Remnants}

Here we summarize the equations governing the evolution of the forward and reverse shock velocities and radii, following Truelove \& McKee (1999). These authors concentrated on the $s=0$ uniform density circumstellar medium case, so here we outline the extension of their results to $s=2$, as implemented by us. The ejecta density profile is taken to be a constant density core with an envelope obeying $\rho(r) \propto r^{-n}$ where $n>5$. The circumstellar medium density profile is $\rho(r) \propto r^{-s}$ where $s<3$. For a uniform density ambient medium $s=0$, and for a steady stellar wind, the case we will take for Cas A, $s=2$. We work in similar units to Truelove \& McKee (1999);

$$
\begin{aligned}
& t_{0}=423 M_{e j}^{5 / 6} E_{51}^{-1 / 2} \rho^{-1 / 3} \text { years } \\
& x_{0}=\quad 3.07 M_{e j}^{1 / 3} \rho^{-1 / 3} \mathrm{pc}
\end{aligned}
$$

for $s=0$ and

$$
\begin{array}{rc}
t_{0}= & 5633 M_{e j}^{3 / 2} E_{51}^{-1 / 2}\left(\rho R_{b}^{2}\right)^{-1} \text { years } \\
x_{0}= & 40.74 M_{e j}\left(\rho R_{b}^{2}\right)^{-1} \mathrm{pc}
\end{array}
$$

for $s=2$, where $M_{e j}$ is the ejecta mass in solar masses, $E_{51}$ is the explosion energy in units of $10^{51} \mathrm{ergs}, \rho$ is the circumstellar density at the blast wave in hydrogen atoms (or equivalent mass) per cc and $R_{b}$ is the blast wave radius in pc. The unit of time $t_{0}$ is related to the so-called Sedov-Taylor time in McKee \& Truelove (1995) by $t_{S T}=t_{0} / 2.024$. The unit of distance is similarly related by a factor 1.377 to their Sedov-Taylor distance $x_{S T}$. These fiducial quantities apply to the case of uniform density ejecta expanding into a uniform density ambient medium. For $t<t_{S T}$ the remnant is in the ejecta dominated phase where the mass of the ejecta is dominant, and for $t>t_{S T}$ the Sedov-Taylor phase where the swept up circumstellar mass dominates the remnant evolution.

The ejecta dominated phase is further divided into an initial period while the reverse shock is propagating through the ejecta envelope, and a later period once it reaches the core. During the initial envelope phase of evolution the blast wave radius is given by (this is the 
generalization of equation (75 of Truelove \& McKee 1999, for arbitrary s)

$$
R_{b}=\left\{\frac{l_{E D}^{(n-2)}}{\phi_{E D}} \frac{3(3-s)^{2}}{4 \pi(n-3) n}\left[\frac{10}{3} \frac{(n-5)}{(n-3)}\right]^{(n-3) / 2}\right\}^{1 /(n-s)} t^{(n-3) /(n-s)} .
$$

Here $l_{E D}$ is the ratio of the radii of the forward shock to the reverse shock, known as the "lead factor", so that $R_{r}=R_{b} / l_{E D}$, and we take $l_{E D}=1.19+8 / n^{2}$. The ratio of the pressures behind the reverse and forward shocks is given by $\phi_{E D}=0.39-0.6 \exp (-n / 4)$. The values for these parameters (both for $s=2$ ) are fitted to the tabulations given in Chevalier (1982). The blast wave velocity is given by

$$
v_{b}=\left(\frac{n-3}{n-s}\right) \frac{R_{b}}{t}
$$

and the reverse shock velocity by

$$
v_{r}=\frac{R_{r}}{t}-\frac{d R_{r}}{d t}=\left(\frac{3-s}{n-3}\right) \frac{v_{b}}{l_{E D}} .
$$

The blast wave radius during the phase when the reverse shock is propagating through the ejecta core is given by (from equation 45 of Truelove \& McKee 1999)

$$
R_{b}^{(3-s) / 2}+\frac{3-s}{3} \sqrt{\frac{l_{E D} f_{0}}{\phi_{E D}}}\left(\frac{R_{b}}{v_{e j} t l_{E D}}\right)^{3 / 2}=(3-s) \sqrt{\frac{l_{E D}}{\phi_{E D}}}\left\{w_{\text {core }}^{3 / 2} f_{0}^{1 / 2} \frac{n}{3(n-3)}-\frac{f_{n}^{1 / 2}}{n-3}\right\}
$$

where $w_{\text {core }}=v_{\text {core }} / v_{e j}$ and $v_{\text {core }}=\sqrt{10 / 3} \sqrt{(n-5) /(n-3)}$ is the ejecta velocity at the core-envelope boundary and $v_{e j}$ is the ejecta velocity at the outer edge of the envelope. In deriving equation A5 we have put $w_{\text {core }} \rightarrow 1$. The ejecta structure function $f(w)=f_{0}$ for $0 \leq w \leq w_{\text {core }}$ and $f(w)=f_{n} / w^{n}$ for $w_{\text {core }} \leq w \leq 1$. From continuity at the core-envelope boundary $f_{0}=f_{n} / w_{\text {core }}^{n} \rightarrow 3 / 4 \pi$ as $w_{\text {core }} \rightarrow 1$. The time $t_{\text {core }}$ where the reverse shock hits the ejecta core is determined from equation A5 by setting $R_{b}=l_{E D} v_{\text {core }} t_{\text {core }}, w_{\text {core }} \rightarrow 1$ so that $v_{\text {core }}=v_{e j}$ and solving for $t_{\text {core }}$ with the result

$$
t_{\text {core }}=\frac{R_{b}}{l_{E D} v_{c o r e}}=\left\{\frac{1}{l_{E D}^{(2-s)} \phi_{E D}} \frac{3(3-s)^{2}}{4 \pi(n-3) n}\right\}^{1 /(3-s)}\left[\frac{3}{10} \frac{(n-3)}{(n-5)}\right]^{1 / 2} .
$$

This generalizes equation 79 of Truelove \& McKee (1999). Equation A8 is quite simple to work with for $s=0$, but for $s=2$ it requires the solution of a cubic equation in $R_{b}^{1 / 2}$, and for arbitrary $s$ can be even more complicated. We adopt a simpler procedure of extending 
the blast wave envelope solution into the core phase and matching it to the offset power law solution that is appropriate in the Sedov-Taylor limit,

$$
v_{b}=\frac{n-3}{n-s} \frac{R_{b}}{t}=\frac{2 \xi_{s}^{1 / 2}}{5-s} R_{b}^{(s-3) / 2}
$$

where $\xi=(5-s)(10-3 s) / 8 \pi$. The time at which these two solutions connect is derived by eliminating $v_{b}$ between equations $\mathrm{A} 6$ and $\mathrm{A} 9$, and then substituting the envelope solution for $R_{b}$, equation $\mathrm{A} 5$ into the resulting expression for $t$ and solving for $t$ with solution

$$
t_{\text {conn }}=\left(\frac{n-3}{n-s} \sqrt{2 \pi \frac{5-s}{10-3 s}}\right)^{2(n-s) /(5-n)(3-s)}\left(v_{e j} l_{E D}\right)^{(n-s)(5-s) /(5-n)(3-s)} t_{\text {core }}^{(5-s) /(5-n)}
$$

The complete expression for the forward shock radius is

$R_{b}=\left\{\left[\left\{\frac{l_{E D}^{n-2} 3(3-s)^{2}}{\phi_{E D} 4 \pi n(n-3)}\right\}\left(t_{c o n n} v_{c o r e}\right)^{n-3}\right]^{(5-s) / 2(n-s)}+\sqrt{\frac{(5-s)(10-3 s)}{8 \pi}}\left(t-t_{c o n n}\right)\right\}^{2 /(5-s)}$

with the velocity given by equation A10.

The reverse shock trajectory through the ejecta core and into the Sedov-Taylor phase cannot in general be specified without recourse to numerical calculations. Truelove \& McKee (1999) show that for $s=0$ SNRs the reverse shock velocity is approximately proportional to time and so introduce a constant acceleration parameterization, with the value of the acceleration being determined numerically. In general the acceleration turns out to be quite small, and the reverse shock velocity is almost constant. By the same methods it can be shown that for $s=2$ SNRs the reverse shock velocity varies as $\sqrt{t}$, and following reverse shock propagation into the ejecta core, we expect it to accelerate much less than in the $s=0$ case, and so during the core-Sedov-Taylor phase we hold $v_{r}$ constant at the value given by equation A7 upon entry into the ejecta core. The reverse shock radius in this phase is given by

$$
R_{r}=\left\{\frac{R_{b}\left(t=t_{\text {core }}\right)}{l_{E D} t_{\text {core }}}-v_{r} \ln \left(t / t_{\text {core }}\right)\right\} t
$$

To summarize, the blast wave radius and velocity are given by equations A45 and A5 for $t<t_{\text {conn }}$, given by equation A11, and are given by equations A10 and A12 for $t>t_{\text {conn }}$. The reverse shock radius and velocity (with respect to the otherwise freely expanding ejecta) are given by equations A5 (divided by $l_{E D}$ ) and A7 for $t<t_{\text {core }}$, with $t_{\text {core }}$ given by equation A9. For $t>t_{\text {core }}$ the reverse shock velocity is held constant and the radius is given by equation A13. 
Truelove \& McKee (1999) give no guidance on the motion of the contact discontinuity. We use the results of Chevalier (1982) for the envelope phase, and during core propagation we assume that the contact discontinuity expands with 0.75 of the forward shock velocity. Detailed calculations indicate that the forward shock-contact discontinuity separation increases once the reverse shock has reached its maximum radius (Wang \& Chevalier 2001). We estimate that this will occur once Cas $\mathrm{A}$ is about 770 years old for the $n=6$ ejecta profile, the with projected time increasing rapidly with increasing $n$. An inconsistency arises with this assumption for the steeper models, in that the reverse shock radius can be ahead of the contact discontinuity. We suspect that our estimate of the reverse shock velocity is becoming inaccurate, and increase $v_{r}$ in these cases so that $R_{r}$ as given by equation $\mathrm{A} 13$ is always less than $R_{c}=0.75 R_{b}$. This modification produces essentially no change in the spectroscopic parameters, $T_{e}$ and $n_{e} t$ in which we are interested. We have also tried several different analytic representations of the hydrodynamics and find our curves of $T_{e}$ against $n_{e} t$ to be insensitive to these differences.

\section{B. BLASPHEMER Simulations}

BLASPHEMER (BLASt Propagation in Highly EMitting EnviRonment) follows the time dependent ionization balance and temperatures of a Lagrangian plasma parcel as it passes through either the forward or reverse shock and then expands with the rest of the supernova remnant. In this appendix we repeat and update some of the description in Laming (2001b). Behind the reverse shock, the density $n_{q}$ of ions with charge $q$ is given by

$$
\frac{d n_{q}}{d t}=n_{e}\left(C_{i o n, q-1} n_{q-1}-C_{i o n, q} n_{q}\right)+n_{e}\left[\left(C_{r r, q+1}+C_{d r, q+1}\right) n_{q+1}-\left(C_{r r, q}+C_{d r, q}\right) n_{q}\right]
$$

where $C_{i o n, q}, C_{r r, q}, C_{d r, q}$ are the rates for electron impact ionization, radiative recombination and dielectronic recombination respectively, out of the charge state $q$. These rates are the same as those used in the recent ionization balance calculations of Mazzotta et al. (1998), using subroutines kindly supplied by Dr P. Mazzotta (private communication 2000). The electron density $n_{e}$ is determined from the condition that the plasma be electrically neutral. The ion and electron temperatures, $T_{i}$ and $T_{e}$ are given by

$$
\frac{d T_{i}}{d t}=-0.13 n_{e} \frac{\left(T_{i}-T_{e}\right)}{A T_{e}^{3 / 2}} \frac{\sum_{q} q^{3} n_{q} /(q+1)}{\sum_{q} n_{q}}
$$

and

$$
\frac{d T_{e}}{d t}=0.13 n_{e} \frac{\left(T_{i}-T_{e}\right)}{A T_{e}^{3 / 2}} \frac{\sum_{q} q^{2} n_{q} /(q+1)}{\sum_{q} n_{q}}-\frac{T_{e}}{n_{e}} \frac{d n_{e}}{d t}-\frac{2}{3 n_{e} k_{\mathrm{B}}} \frac{d Q}{d t} .
$$


Here $A$ is the atomic mass of the ions in the plasma. The last term $d Q / d T$ represents plasma energy losses due to ionization and radiation. Radiation losses are taken from Summers \& McWhirter (1979). At each time step $T_{i}$ and $T_{e}$ are modified by a further factor $\exp \left(-4 v_{e x} \Delta t / 3 r\right)$ and $n_{e}$ and the $n_{q}$ by $\exp \left(-2 v_{e x} \Delta t / r\right)$. Here $v_{e x}$ is the expansion velocity, which is assumed constant for all ejecta, and is given by the expansion velocity of the contact discontinuity discussed above. The radial compression/decompression of shells of shocked ejecta is treated by further modifying densities and temperatures by $v_{e x} / v_{e x}^{\prime}$ and $\left(v_{e x} / v_{e x}^{\prime}\right)^{2 / 3}$ respectively at each time step, where $v_{e x}^{\prime}$ is $v_{e x}$ at the previous time step. The plasma pressure is evolved according to adiabatic expansion in the same way, and the densities and temperatures further adjusted by $\left(P / P^{\prime}\right)^{a}$ where $a=0.6$ for densities and 0.4 for temperatures. This accounts for the compression of plasma due to radiation and ionization losses. The initial plasma pressure is $P$ and the plasma pressure after losses is $P^{\prime}$, and the Lagrangian plasma element is recompressed adiabatically at each time step to restore its pressure to the adiabatic value after losses. Except for ejecta near the contact discontinuity, radiation losses are generally unimportant.

Spitzer (1978) gives the timescale for an electron distribution to relax to a Maxwellian as

$$
t_{e q}(e, e)=\frac{3 m_{e}^{1 / 2}\left(k_{\mathrm{B}} T_{e}\right)^{3 / 2}}{8 \sqrt{2 \pi} n_{e} e^{4} \ln \Lambda}
$$

where $\Lambda$ is the so-called plasma parameter, the ratio of largest to smallest impact parameters for collisions. In supernova remnants $\ln \Lambda \simeq 40$. The equilibration time for ions $t_{e q}(i, i)=$ $t_{e q}(e, e) \sqrt{m_{i} / m_{e}} / Z_{i}^{4}$, and that for electron-ion equilibration is $t_{e q}(e, i)=t_{e q}(e, e) m_{i} / m_{e} / Z_{i}^{2}$ where $Z_{i}$ is the ion charge. Accordingly we write

$$
\frac{d \Delta T}{d t}=-0.13 Z^{2} n_{e} \frac{\Delta T}{A T_{e}^{3 / 2}}
$$

which is equation (2), with $\Delta T=T_{i}-T_{e}$. We consider a fully ionized gas with $n_{e}=Z n_{i}$ and

$$
\frac{d}{d t}\left(n_{i} T_{i}+n_{e} T_{e}\right)=n_{i} \frac{d T_{i}}{d t}+n_{e} \frac{d T_{e}}{d t}=0 .
$$

Solving these equations yields

$$
\begin{gathered}
\frac{d T_{e}}{d t}=0.13 \frac{Z^{2} n_{e}}{Z+1} \frac{T_{i}-T_{e}}{A T_{e}^{3 / 2}} \\
\frac{d T_{i}}{d t}=-0.13 \frac{Z^{3} n_{e}}{Z+1} \frac{T_{i}-T_{e}}{A T_{e}^{3 / 2}} .
\end{gathered}
$$

In deriving equations (14) and (15) these expressions are averaged over the ion charge states in the plasma, and the expression for $d T_{e} / d t$ is modified by the inclusion of terms accounting 
@PJL ENTER LANGUAGE=POSTSCRIPT

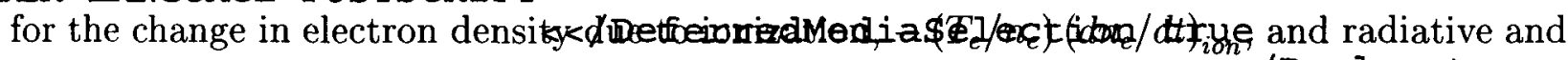
ionization losses, $-\left(2 / 3 n_{e} k_{\mathrm{B}}\right) d Q / d t$. Recombinations, which reduce the electrotedentity not result in an increase in the electron temperature in low density plasmas, since the energy of the recombined electron is radiated away, rather than being shared with the other plasma electrons as would be the case for three-body recombination in dense plasmas.

\section{REFERENCES}

Aharonian, F. et al. 2001, A\&A, 370, 112

Akiyama, S., Wheeler, J. C., Meier, D. L., \& Lichenstadt, I. 2003, ApJ, 584, 954

Allende Prieto, C., Lambert, D. L., \& Asplund, M. 2001, ApJ, 556, L63

Allende Prieto, C., Lambert, D. L., \& Asplund, M. 2002, ApJ, 573, L137

Anders, E., \& Grevesse, N. 1989, Geochim. Cosmochim. Acta, 53, 197

Anderson, M., \& Rudnick, L. 1995, ApJ, 441, 307

Ashworth, W. B. 1980, J. Hist. Astron., 11, 1

Berezhko, E. G., Pühlhofer, G., \& Völk, H. J. 2003, A\&A, 400, 971

Bleeker, J. A. M., Willingale, R., van der Heyden, K., Dennerl, K., Kaastra, J. S., Aschenbach, B., \& Vink, J. 2001, A\&A, 365, L225

Blondin, J. M., Borkowski, K. J., \& Reynolds, S. P. 2001, ApJ, 557, 782

Blondin, J. M., \& Ellison, D. C. 2001, 560, 244

Borkowski, K. J., Szymkowiak, A. E., Blondin, J. M., \& Sarazin, C. L. 1996, ApJ, 466, 866

Chevalier, R. A., \& Fransson, C. 1994, ApJ, 420, 268

Chevalier, R. A. 1982, ApJ, 258, 790

Chevalier, R. A., \& Kirshner, R. P. 1979, ApJ, 233, 154

Delaney, T. A., \& Rudnick, L. 2003, ApJ, in press, astro-ph/0303399

Fabian, A. C., Willingale, R., Pye, J. P., Murray, S. S., \& Fabbiano, G. 1980, MNRAS, 193, 175 
Favata, F., Vink, J., Dal Fiume, D., Parmar, A. N., Santangelo, A., Mineo, T., PreiteMartinez, A., Kaastra, J. S., \& Bleeker, J. A. M. 1997, A\&A, 324, L49

Fesen, R. A. 2001, ApJS, 133, 161

Fryer, C. L., \& Warren, M. S. 2002, ApJ, 574, L65

Fryer, C. L., \& Warren, M. S. 2003, in preparation

Fryer, C. L., \& Heger, A. 2000, ApJ, 541, 1033

Garcia-Segura, G., Langer, N., \& Mac Low, M.-M. 1996, A\&A, 316, 133

Gotthelf, E. V., Koralesky, B., Rudnick, L., Jones, T. W., Hwang, U., \& Petre, R. 2001, ApJ552, L39

Grevess, N., \& Sauval, A. J. 1998, Space Science Reviews, 85, 161

Holweger, H. 2001 in AIP Conference Proceedings 598, Solar and Galactic Composition, ed. R. F. Wimmer-Schweingruber, p23

Hwang, U., \& Laming, J. M. 2003, ApJ, submitted

Iyudin, A. F., et al. 1994, A\&A, 284, L1

Iyudin, A. F., Diehl, R., Lichti, G. G., et al. 1997, ESA SP-382, 37

Kamper, K., \& van den Bergh, S. 1976, ApJS, 32, 351

Khokhlov, A. M., Höflich, P. A., Oran, E. S., Wheeler, J. C., Wang, L., \& Chtchelkanova, A. Yu. 1999, ApJ, 524, L107

Klein, R. I., McKee, C. F., \& Colella, P. 1994, ApJ, 420, 213

Klein, R. I., Budil, K. S., Perry, T. S., \& Bach, D. R. 2003, ApJ, 583, 245

Koralesky, B., Rudnick, L., Gotthelf, E. V., \& Keohane, J. W. 1998, ApJ, 505, L27

Kassim, N. E., Perley, R. A., Dwarakanath, K. S., \& Erickson, W. C. 1995, ApJ, 455, L59

Lamers, H. J. G. L. M., \& Nugis, T. 2002, A\&A, 395, L1

Laming, J. M. 2001a, ApJ, 546, 1149

Laming, J. M. 2001b, ApJ, 563, 828 
Laming, J. M., \& Grun, J. 2002, Phys. Rev. Lett., 89, 125002

Laming, J. M., \& Grun, J. 2003, Physics of Plasmas, in press

Massey, P., DeGioia-Eastwood, K., \& Waterhouse, E. 2001, AJ, 121, 1050

Mazzotta, P., Mazzitelli, G., Colafranceso, S., \& Vittorio, N. 1998, A\&AS, 133, 403

McKee, C. F., \& Cowie, L. L. 1975, ApJ, 195, 715

McKee, C. F., \& Truelove, J. K. 1995, Phys. Rep., 256, 157

Nagataki, S., Hashimoto, M., Sato, K., Yamada, S., \& Mochizuki, Y. 1998, ApJ, 492, L45

Poludnenko, A. Y., Frank, A., \& Blackman, E. G. 2001, ApJ, 576, 832

Reber, G. 1944, ApJ, 100, 279

Reed, J. E., Hester, J. J., Fabian, A. C., \& Winkler, P. F. 1995, ApJ, 440, 706

Reichart, D. E., \& Stephens, A. W. 2000, ApJ, 537, 904

Rothschild, R. E., \& Lingenfelter, R. E. 2003, ApJ, 582, 257

Ryle, M., \& Smith, F. G. 1948, Nature, 162, 462

Sgro, A. 1975, ApJ, 197, 621

Spitzer, L. Jr., Physical Processes in the Interstellar Medium, (New York: Wiley)

Summers, H. P., \& McWhirter, R. W. P., J. Phys. B., 12, 2387

Sutherland, R. S., \& Dopita, M. A. 1995, ApJ, 439, 381

Thorstensen, J. R., Fesen, R. A., \& van den Bergh, S. 2001, AJ, 122, 297

Timmes, F. X., Woosley, S. E., Hartmann, D. H., \& Hoffman, R. D. 1996, ApJ, 464, 332

Truelove, J. K., \& McKee, C. F. 1999, ApJS, 120, 299

van den Bergh, S., \& Kamper, K. 1985, ApJ, 293, 537

van den Bergh, S. 1971, ApJ, 165, 457

Vink, J., \& Laming, J. M. 2003, ApJ, 584, 758 
Vink, J., Laming, J. M., Kaastra, J. S., Bleeker, J. A. M., Bloemen, H., \& Oberlack, U. 2001, ApJ, 560, L79

Vink, J., Bloemen, H., Kaastra, J. S., \& Bleeker, J. A. M. 1998, A\&A, 339, 201

Vink, J., Kaastra, J. S., \& Bleeker, J. A. M. 1996, A\&A, 307, L41

Wang, C.-Y., \& Chevalier, R. A. 2001, ApJ, 549, 1119

Wang, C.-Y., \& Chevalier, R. A. 2002, ApJ, 574, 155

Willingale, R., Bleeker, J. A. M., van der Heyden, K. J \& Kaastra, J. S. 2003, A\&A, 398, 1021

Woosley, S. E., Langer, N., \& Weaver, T. A. 1993, ApJ, 411, 823 


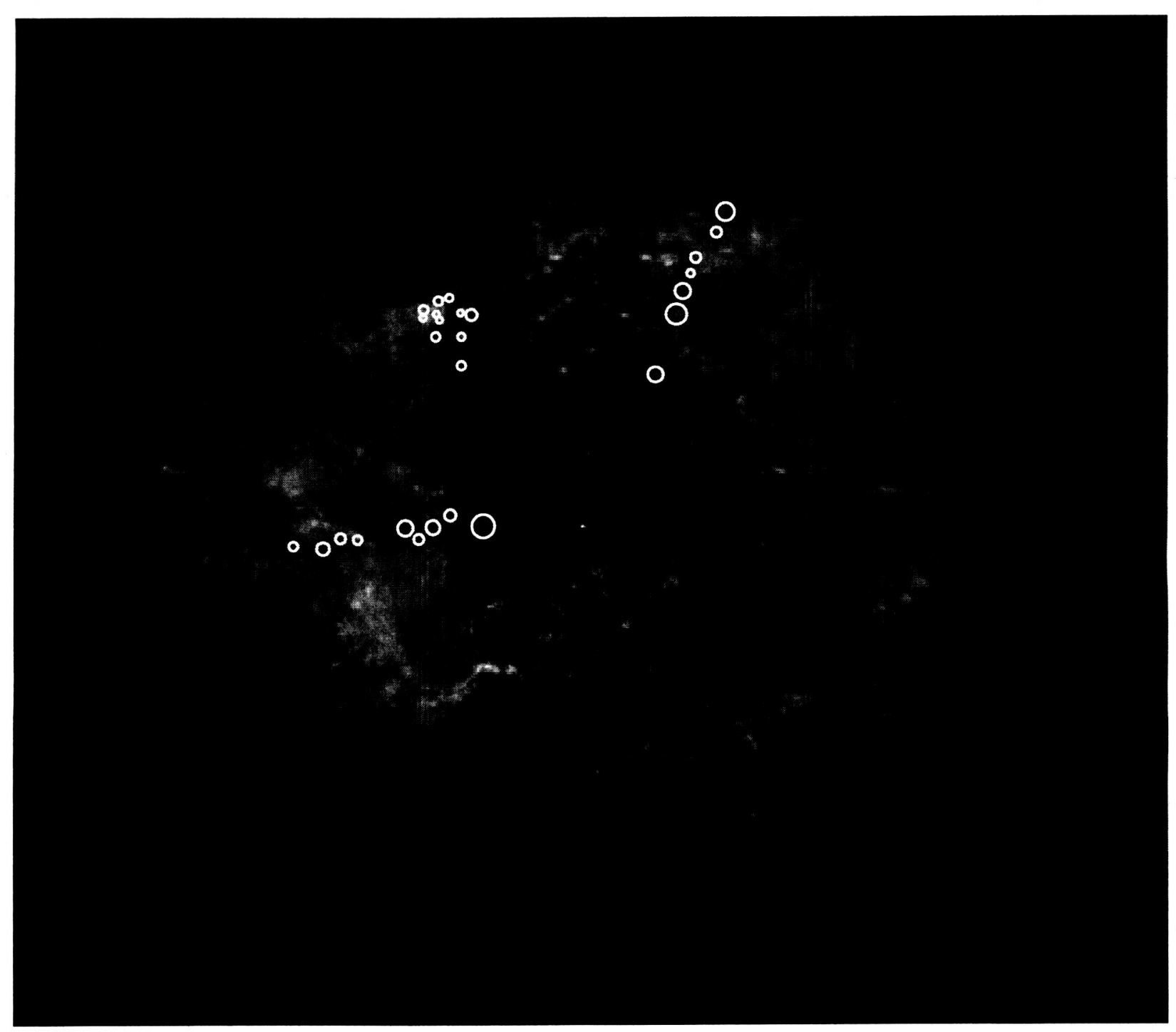

Fig. 1.- Locations of series of "Si" rich knots on northern limb. East is to the left and north is up. 


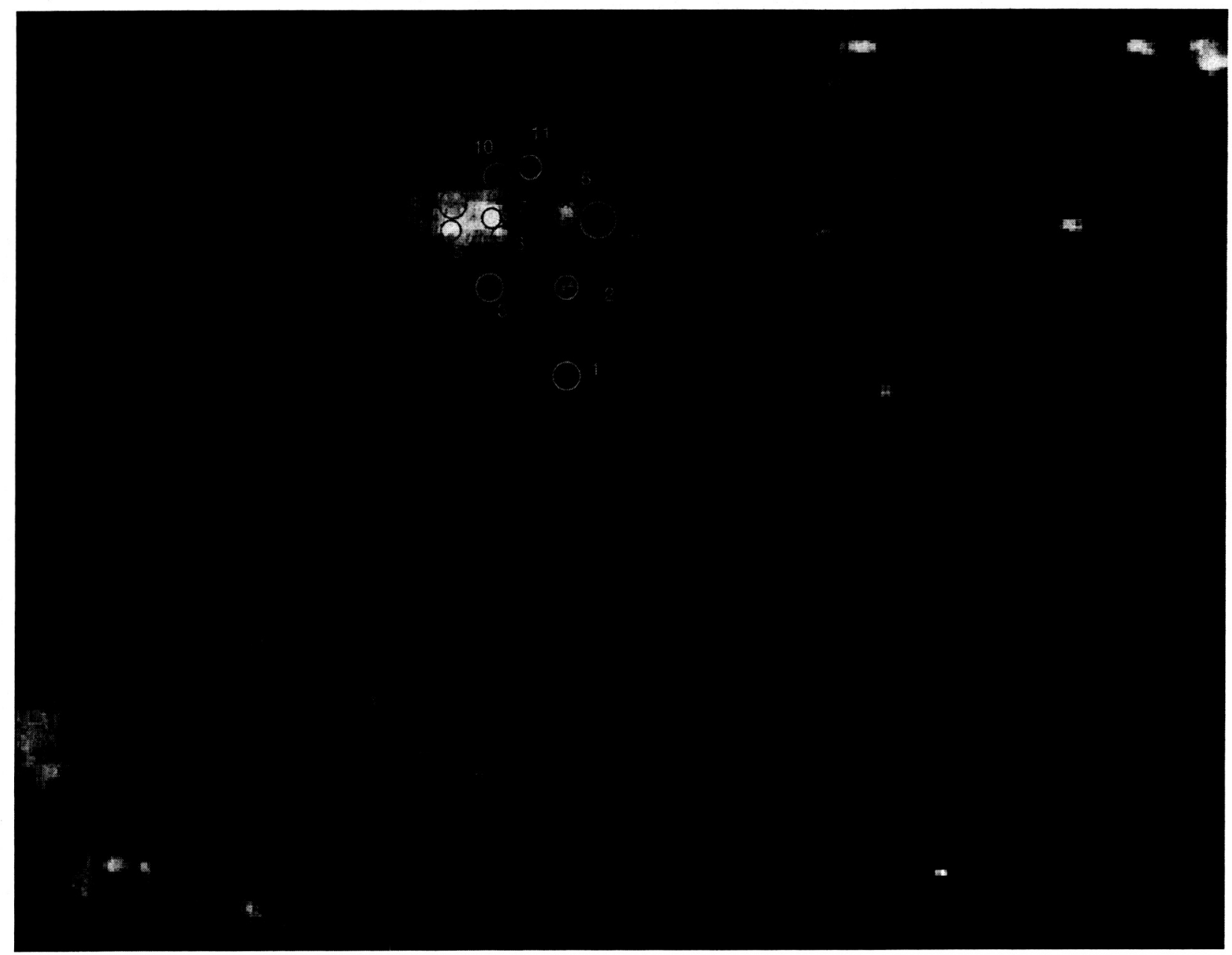

Fig. 2.- Detail of Cas A showing locations of "Si" rich knots at the jet base in the NE region. East is to the left and north is up. 


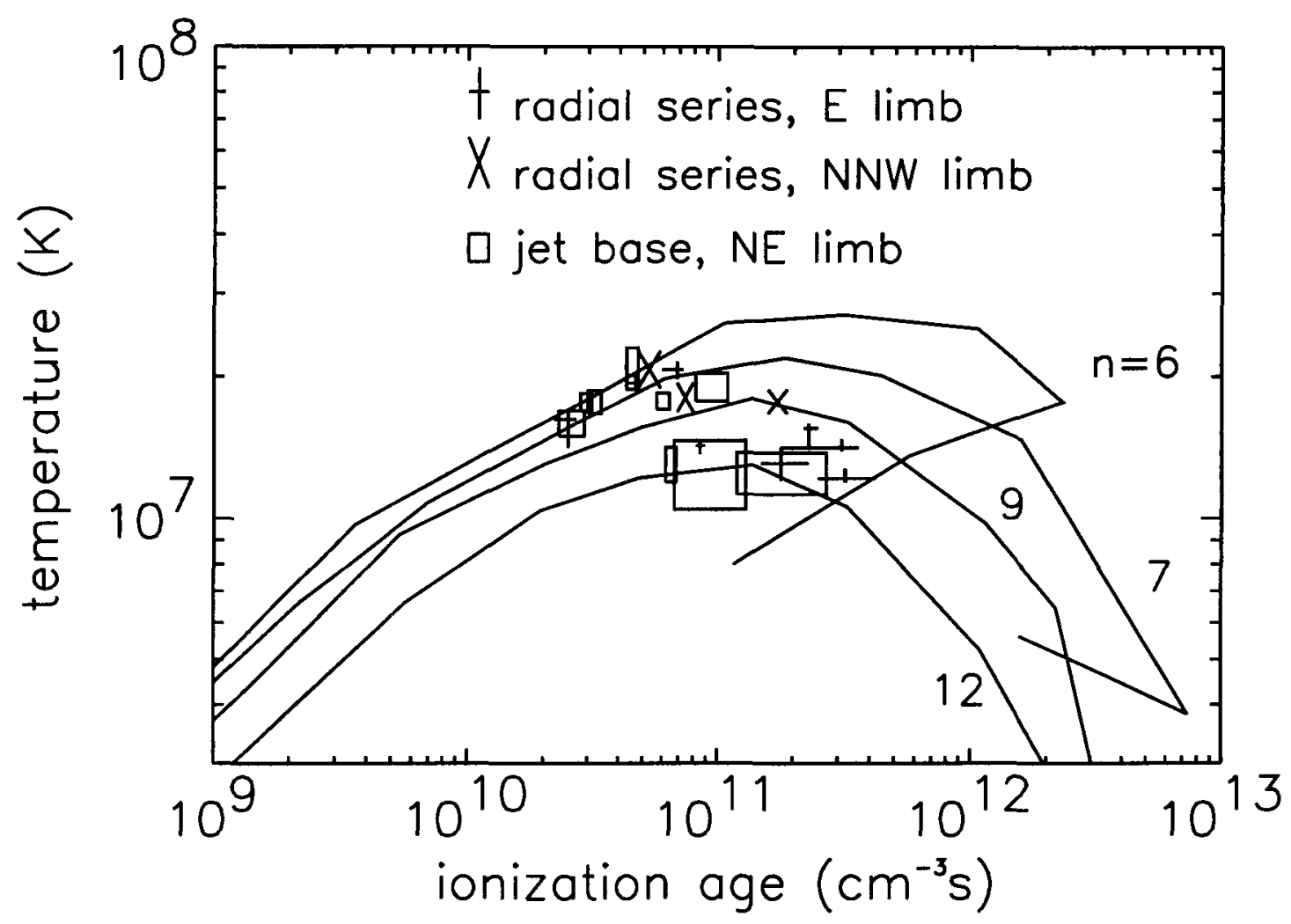

Fig. 5.- Plots of $T_{e}$ against $n_{e} t$ for varying ejecta envelope power laws. The solid lines give models with $M_{e j}=2 M_{\odot}, E_{51}=3$ and $\rho r_{b}^{2}=21$, for $n=5,5,6,7,9$, and 12 from the top. The point at highest $n_{e} t$ for $n=5.5$ and 6 corresponds to ejecta at the core-envelope boundary. For higher values of $n$ this plasma undergoes thermal instability. The dotted lines indicate the variation of $T_{e}$ with $n_{e} t$ for the modified models in Table 4 for $n=5.5,6$. Higher values of $n$ change insignificantly in the modified models. Only fits with $\chi^{2} /$ d.o.f. $<2$ are plotted. 


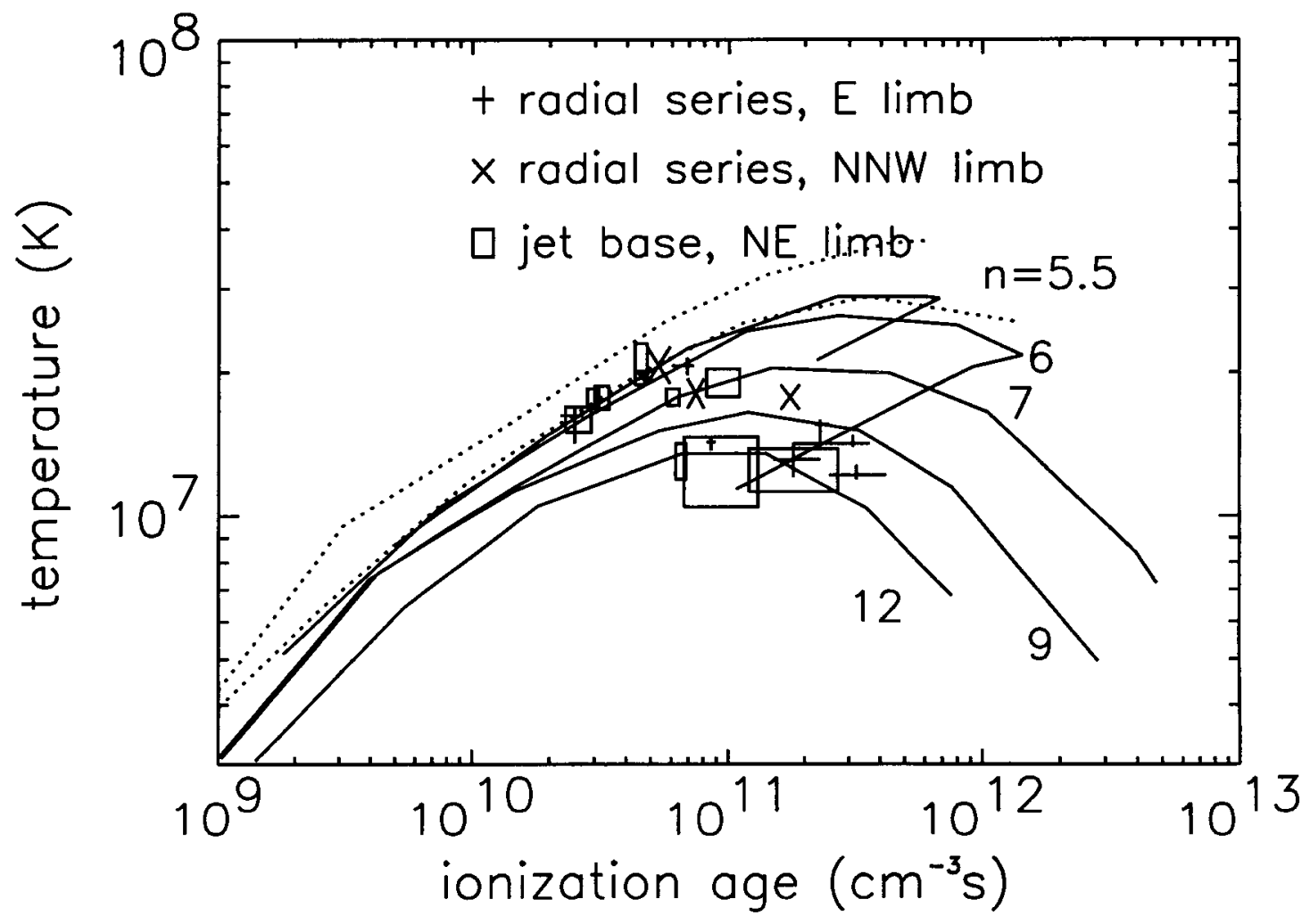

Fig. 6.- Plots of $T_{e}$ against $n_{e} t$ for varying ejecta envelope power laws. The solid lines give models with $M_{e j}=2 M_{\odot}, E_{51}=2$ and $\rho r_{b}^{2}=14$, for $n=5.5,6,7,9$, and 12 from the top. The point at highest $n_{e} t$ for $n=5.5,6$, and 7 corresponds to ejecta at the core-envelope boundary. For higher values of $n$ this plasma undergoes thermal instability. The dotted lines indicate the variation of $T_{e}$ with $n_{e} t$ for the modified models in Table 5 for $n=5.5,6$. Higher values of $n$ change insignificantly in the modified models. Only fits with $\chi^{2} /$ d.o.f. $<2$ are plotted. 
Table 1. Fits to O-Si rich Knots

\begin{tabular}{|c|c|c|c|c|c|c|c|c|c|}
\hline $\operatorname{knot}^{\mathrm{a}}$ & $k_{\mathrm{B}} T_{e}(\mathrm{keV})^{\mathrm{b}}$ & $n_{e} t\left(\mathrm{~cm}^{-3} \mathrm{~s}\right)$ & $\mathrm{Si} / \mathrm{O}^{\mathrm{c}}$ & $\mathrm{Fe} / \mathrm{O}$ & $n_{\mathrm{H}}\left(10^{22} \mathrm{~cm}^{-2}\right)$ & $\chi^{2}$ & $\chi^{2} /$ dof & no. dof & no. cts \\
\hline \multirow[t]{2}{*}{$\mathrm{E} 1$} & 1.78 & $6.9 e+10$ & 0.08 & 0.05 & 1.60 & 233.1 & 1.46 & 160 & 9832 \\
\hline & $1.70-1.85$ & $6.0 e+10-7.3 e+10$ & $0.07-0.09$ & $0.04-0.06$ & $1.49-1.67$ & & & & \\
\hline \multirow[t]{2}{*}{$\mathrm{E} 2$} & 1.40 & $2.5 \mathrm{e}+10$ & 0.35 & 0.04 & 1.99 & 199.9 & 1.54 & 130 & 7976 \\
\hline & $1.22-1.47$ & $2.2 e+10-2.7 e+10$ & $0.31-0.37$ & $0.03-0.04$ & $1.96-2.05$ & & & & \\
\hline \multirow[t]{2}{*}{ E3 } & 1.05 & $3.2 \mathrm{e}+11$ & 0.28 & 0.17 & 1.22 & 236.7 & 1.75 & 135 & 11897 \\
\hline & $1.03-1.10$ & $2.5 \mathrm{e}+11-4.2 \mathrm{e}+11$ & $0.24-0.39$ & $0.13-0.20$ & $1.16-1.28$ & & & & \\
\hline \multirow[t]{2}{*}{$\mathrm{E} 4$} & 1.13 & $1.8 \mathrm{e}+11$ & 0.29 & 0.07 & 1.11 & 184.7 & 1.71 & 108 & 5994 \\
\hline & $1.04-1.22$ & $1.5 e+11-2.3 e+11$ & $0.27-0.35$ & $0.06-0.10$ & $1.03-1.24$ & & & & \\
\hline \multirow[t]{2}{*}{ E5 } & 1.34 & $2.3 e+11$ & 0.29 & 0.12 & 0.99 & 236.9 & 1.74 & 136 & 10797 \\
\hline & $1.21-1.37$ & $2.2 e+11-2.5 e+11$ & $0.27-0.31$ & $0.09-0.12$ & $0.96-1.02$ & & & & \\
\hline \multirow[t]{2}{*}{ E6 } & 1.23 & $8.5 e+10$ & 0.29 & 0.05 & 1.51 & 271.8 & 1.84 & 148 & 10452 \\
\hline & $1.18-1.25$ & $8.2 e+10-8.9 e+10$ & $0.28-0.34$ & $0.04-0.06$ & $1.49-1.58$ & & & & \\
\hline E7 & 0.91 & $2.0 \mathrm{e}+11$ & 0.28 & 0.04 & 1.2 & 332.8 & 2.47 & 135 & 9389 \\
\hline E8 & 0.72 & $4.2 e+11$ & 0.45 & 0.04 & 1.2 & 296.0 & 2.29 & 129 & 10316 \\
\hline \multirow[t]{2}{*}{ E9 } & 1.22 & $3.1 \mathrm{e}+11$ & 0.28 & 0.13 & 1.20 & 163.6 & 1.56 & 105 & 4924 \\
\hline & $1.20-1.27$ & $1.8 e+11-3.6 e+11$ & $0.23-0.36$ & $0.11-0.21$ & $1.17-1.28$ & & & & \\
\hline NNW1 & 1.42 & $1.1 \mathrm{e}+11$ & 0.29 & 0.12 & 1.3 & 335.8 & 2.05 & 164 & 15126 \\
\hline NNW2 & 1.85 & $1.2 \mathrm{e}+11$ & 0.24 & 0.11 & 1.1 & 760.7 & 3.21 & 237 & 32844 \\
\hline NNW3 & 1.75 & $1.4 \mathrm{e}+11$ & 0.21 & 0.09 & 1.0 & 548.1 & 3.11 & 176 & 16483 \\
\hline \multirow[t]{2}{*}{ NNW4 } & 1.86 & $5.1 e+10$ & 0.32 & 0.11 & 1.49 & 203.3 & 1.42 & 143 & 8725 \\
\hline & $1.63-1.95$ & $4.8 e+10-5.9 e+10$ & $0.29-0.34$ & $0.10-0.12$ & $1.45-1.54$ & & & & \\
\hline \multirow[t]{2}{*}{ NNW5 } & 1.49 & $7.6 \mathrm{e}+10$ & 0.24 & 0.08 & 1.22 & 242.9 & 1.48 & 164 & 12960 \\
\hline & $1.45-1.67$ & $6.9 e+10-8.0 e+10$ & $0.22-0.25$ & $0.07-0.09$ & $1.20-1.28$ & & & & \\
\hline NNW6 & 1.10 & $3.3 e+10$ & 0.20 & 0.00 & 1.7 & 305.0 & 2.18 & 140 & 10584 \\
\hline \multirow[t]{2}{*}{ NNW7 } & 1.54 & $1.7 \mathrm{e}+11$ & 0.18 & 0.04 & 0.84 & 210.9 & 1.70 & 124 & 6826 \\
\hline & $1.44-1.62$ & $1.6 \mathrm{e}+11-1.9 \mathrm{e}+11$ & $0.17-0.19$ & $0.03-0.04$ & $0.76-0.86$ & & & & \\
\hline \multirow[t]{2}{*}{ NE1 } & 1.11 & $1.6 \mathrm{e}+11$ & 0.06 & 0.08 & 1.08 & 87.4 & 1.00 & 87 & 4124 \\
\hline & $0.97-1.19$ & $1.2 \mathrm{e}+11-2.7 \mathrm{e}+11$ & $0.05-0.08$ & $0.06-0.10$ & $0.95-1.24$ & & & & \\
\hline \multirow[t]{2}{*}{ NE2 } & 1.12 & $6.5 e+10$ & 0.40 & 0.04 & 1.24 & 189.1 & 1.70 & 111 & 6009 \\
\hline & $1.03-1.22$ & $6.2 \mathrm{e}+10-6.8 \mathrm{e}+10$ & $0.38-0.52$ & $0.03-0.04$ & $1.10-1.29$ & & & & \\
\hline \multirow[t]{2}{*}{ NE3 } & 1.54 & $3.2 \mathrm{e}+10$ & 0.29 & 0.08 & 1.35 & 119.6 & 1.34 & 90 & 4155 \\
\hline & $1.44-1.61$ & $3.0 \mathrm{e}+10-3.4 \mathrm{e}+10$ & $0.27-0.31$ & $0.07-0.09$ & $1.32-1.37$ & & & & \\
\hline \multirow[t]{2}{*}{ NE4 } & 1.53 & $3.0 \mathrm{e}+10$ & 0.60 & 0.04 & 1.29 & 266.8 & 1.89 & 141 & 10884 \\
\hline & $1.46-1.59$ & $2.8 \mathrm{e}+10-3.1 \mathrm{e}+10$ & $0.57-0.62$ & $0.03-0.04$ & $1.27-1.33$ & & & & \\
\hline NE5 & 1.51 & $3.3 e+10$ & 0.44 & 0.03 & 1.4 & 284.1 & 2.47 & 115 & 6961 \\
\hline \multirow[t]{2}{*}{ NE6 } & 1.32 & $2.8 \mathrm{e}+10$ & 0.34 & 0.00 & 1.61 & 191.4 & 1.48 & 129 & 7578 \\
\hline & $1.29-1.46$ & $2.3 e+10-2.9 e+10$ & $0.32-0.36$ & $0.00-0.003$ & $1.60-1.63$ & & & & \\
\hline \multirow[t]{2}{*}{ NE7 } & 1.67 & $4.5 e+10$ & 0.25 & 0.06 & 1.54 & 280.4 & 1.76 & 159 & 10016 \\
\hline & $1.62-1.73$ & $4.3 e+10-4.7 e+10$ & $0.24-0.26$ & $0.05-0.06$ & $1.52-1.56$ & & & & \\
\hline \multirow[t]{2}{*}{ NE8 } & 1.90 & $4.3 e+10$ & 0.26 & 0.04 & 1.52 & 248.0 & 1.53 & 162 & 9776 \\
\hline & $1.67-1.98$ & $4.3 e+10-4.8 e+10$ & $0.23-0.27$ & $0.03-0.04$ & $1.48-1.56$ & & & & \\
\hline \multirow[t]{2}{*}{ NE9 } & 1.51 & $6.1 e+10$ & 0.21 & 0.05 & 1.31 & 308.2 & 1.78 & 173 & 14081 \\
\hline & $1.47-1.59$ & $5.7 e+10-6.4 e+10$ & $0.19-0.22$ & $0.04-0.05$ & $1.28-1.32$ & & & & \\
\hline \multirow[t]{2}{*}{ NE10 } & 1.67 & $9.1 e+10$ & 0.06 & 0.09 & 1.31 & 221.9 & 1.66 & 134 & 8451 \\
\hline & $1.53-1.75$ & $8.2 \mathrm{e}+10-1.1 \mathrm{e}+11$ & $0.05-0.08$ & $0.07-0.10$ & $1.27-1.38$ & & & & \\
\hline NE11 & 1.10 & $9.1 e+10$ & 0.12 & 0.17 & 1.32 & 162.7 & 1.75 & 93 & 4812 \\
\hline
\end{tabular}


Table 1-Continued

\begin{tabular}{ccccccccc}
\hline \hline $\mathrm{knot}^{\mathrm{a}}$ & $k_{\mathrm{B}} T_{e}(\mathrm{keV})^{\mathrm{b}}$ & $n_{e} t\left(\mathrm{~cm}^{-3} \mathrm{~s}\right)$ & $\mathrm{Si} / \mathrm{O}^{\mathrm{c}}$ & $\mathrm{Fe} / \mathrm{O}$ & $n_{\mathrm{H}}\left(10^{22} \mathrm{~cm}^{-2}\right)$ & $\chi^{2}$ & $\chi^{2} /$ dof no. dof no. cts \\
\hline $0.90-1.26$ & $6.7 \mathrm{e}+10-1.3 \mathrm{e}+11$ & $0.09-0.15$ & $0.14-0.21$ & $1.22-1.41$ & & \\
\hline
\end{tabular}

${ }^{a}$ Knots are numbered in the $\mathrm{E}$ and NNW regions starting with the innermost and working out to the limb. The knot numbering in the NNE region is given in Figure 2.

bElectron temperature in keV.

${ }^{c}$ Element abundance ratio by number relative to solar values of Anders \& Grevesse (1989). Note that these are superseded by Grevesse \& Sauval (1998), Allende Prieto, Lambert, \& Asplund (2001), and Allende Prieto, Lambert, \& Asplund (2002). In particular abundances relative to $O$ increase by 1.75 .

Table 2. Cas A Ejecta Profile Models $M_{e j}=2 M_{\odot}, E_{51}=3, \rho r_{b}^{2}=21$

\begin{tabular}{|c|c|c|c|c|c|c|c|c|c|c|}
\hline$n$ & $\begin{array}{c}v_{b}(320 \mathrm{yrs}) \\
\mathrm{km} \mathrm{s}^{-1}\end{array}$ & $\begin{array}{c}r_{b}(320 \mathrm{yrs}) \\
\mathrm{pc}\end{array}$ & $\eta^{\mathrm{a}}$ & $\begin{array}{c}r_{r}(320 \mathrm{yrs}) \\
\mathrm{pc}\end{array}$ & $r_{b} / r_{r}$ & $\begin{array}{r}v_{\text {core }}{ }^{b} \\
\mathrm{~km} \mathrm{~s}^{-1}\end{array}$ & $\begin{array}{c}t_{\text {core }}{ }^{c} \\
\text { yrs }\end{array}$ & $\begin{array}{c}t_{c o n n}{ }^{d} \\
y r s\end{array}$ & $\begin{array}{c}t_{\text {rad }} \\
\text { yrs }\end{array}$ & $\begin{array}{l}M_{\text {rad }} \mathrm{f} \\
M_{\odot}\end{array}$ \\
\hline 5.5 & 4044 & 1.85 & 0.72 & 0.92 & 2.00 & 7098 & 39.1 & 50586 & - & 0 \\
\hline 6 & 4942 & 2.15 & 0.75 & 0.97 & 2.21 & 9163 & 21.5 & 632 & - & 0 \\
\hline 7 & 5139 & 2.36 & 0.71 & 1.13 & 2.08 & 11223 & 10.1 & 120.2 & $10.05-10.25$ & 0.012 \\
\hline 8 & 5108 & 2.38 & 0.70 & 1.35 & 1.77 & 12294 & 5.99 & 72.4 & $4.55-10.4$ & 0.41 \\
\hline 9 & 5095 & 2.40 & 0.70 & 1.58 & 1.52 & 12959 & 3.97 & 55.0 & $2.67-11.1$ & 0.61 \\
\hline 10 & 5088 & 2.41 & 0.69 & 1.79 & 1.34 & 13414 & 2.84 & 45.7 & $1.76-11.4$ & 0.69 \\
\hline 11 & 5083 & 2.41 & 0.69 & 1.99 & 1.21 & 13745 & 2.14 & 39.8 & $1.25-11.9$ & 0.75 \\
\hline 12 & 5079 & 2.41 & 0.69 & 2.17 & 1.11 & 13997 & 1.67 & 35.6 & $0.93-12.5$ & 0.79 \\
\hline
\end{tabular}

${ }^{a}$ Forward shock expansion parameter.

${ }^{b}$ Free expansion velocity of ejecta core-envelope boundary.

${ }^{c}$ Time following explosion when reverse shock enters ejecta core.

${ }^{d}$ Time when blast wave solutions are connected.

${ }^{\text {e}}$ Time interval for which ejecta passing through the reverse shock cools to optically emitting temperatures within 320 years.

${ }^{\mathrm{f}}$ Mass of gas that can cool to optically emitting temperatures within 320 years of explosion. 
Table 3. Cas A Ejecta Profile Models $M_{e j}=2 M_{\odot}, E_{51}=2, \rho r_{b}^{2}=14$

\begin{tabular}{cccccccccccc}
\hline$n$ & $\begin{array}{c}v_{b}(320 \mathrm{yrs}) \\
\mathrm{km} \mathrm{s}^{-1}\end{array}$ & $\begin{array}{c}r_{b}(320 \mathrm{yrs}) \\
\mathrm{pc}\end{array}$ & $\eta^{\mathrm{a}}$ & $\begin{array}{c}r_{r}(320 \mathrm{yrs}) \\
\mathrm{pc}\end{array}$ & $r_{b} / r_{r}$ & $\begin{array}{c}v_{\text {core }} \mathrm{b} \\
\mathrm{km} \mathrm{s}^{-1}\end{array}$ & $\begin{array}{c}t_{\text {core }}^{\mathrm{c}} \\
\mathrm{yrs}\end{array}$ & $\begin{array}{c}t_{\text {conn }} \mathrm{d}^{\mathrm{yrs}} \\
\mathrm{yrs}\end{array}$ & $\begin{array}{c}t_{\text {rad }} \mathrm{e}^{\mathrm{e}} \\
\mathrm{yrs}\end{array}$ & $\begin{array}{c}M_{\text {rad }} \\
M_{\odot}\end{array}$ \\
\hline 5.5 & 3928 & 1.79 & 0.72 & 1.08 & 1.66 & 5795 & 71.8 & 92930 & - & 0 \\
6 & 4698 & 2.04 & 0.75 & 1.17 & 1.75 & 7482 & 39.5 & 1162 & - & 0 \\
7 & 5239 & 2.27 & 0.76 & 1.29 & 1.76 & 9163 & 18.6 & 221 & - & 0 \\
8 & 5177 & 2.32 & 0.73 & 1.44 & 1.62 & 10038 & 11.0 & 133 & $9.25-15.5$ & 0.26 \\
9 & 5153 & 2.35 & 0.72 & 1.59 & 1.48 & 10581 & 7.3 & 101 & $5.35-16.5$ & 0.50 \\
10 & 5139 & 2.36 & 0.71 & 1.74 & 1.36 & 10953 & 5.2 & 84.0 & $3.45-17$ & 0.60 \\
11 & 5129 & 2.37 & 0.71 & 1.87 & 1.27 & 11223 & 3.93 & 73.1 & $2.45-17.5$ & 0.66 \\
12 & 5121 & 2.37 & 0.71 & 2.00 & 1.19 & 11429 & 3.07 & 65.5 & $1.815-18$ & 0.70 \\
\hline
\end{tabular}

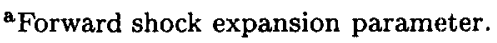

${ }^{b}$ Free expansion velocity of ejecta core-envelope boundary.

c Time following explosion when reverse shock enters ejecta core.

dTime when blast wave solutions are connected.

'Time interval for which ejecta passing through the reverse shock cools to optically emitting temperatures within 320 years.

${ }^{\mathrm{f}}$ Mass of gas that can cool to optically emitting temperatures within 320 years of explosion.

Table 4. Models $r_{b}=2.40 \mathrm{pc}, \rho_{\text {core }}=1.22 e 6 \mathrm{~g} \mathrm{~cm}^{-3} \mathrm{~s}^{3}$, and $\rho r_{b}^{2}=21 \mathrm{H}$ atom $\mathrm{cm}^{-3} \mathrm{pc}^{2}$

\begin{tabular}{ccccccccc}
\hline \hline$n$ & $\begin{array}{c}M_{e j} \\
M_{\odot}\end{array}$ & $\begin{array}{c}E_{51} \\
10^{51} \mathrm{ergs}\end{array}$ & $\begin{array}{c}v_{b}(320 \mathrm{yrs}) \\
\mathrm{km} \mathrm{s}^{-1}\end{array}$ & $\begin{array}{c}r_{b}(320 \mathrm{yrs}) \\
\mathrm{pc}\end{array}$ & $\eta^{\mathrm{a}}$ & $\begin{array}{c}r_{r}(320 \mathrm{yrs}) \\
\mathrm{pc}\end{array}$ & $r_{b} / r_{r}$ & $\begin{array}{c}M_{e j}(n-3) / n / v_{\text {core }}^{3} \mathrm{~b} \\
\mathrm{~g} \mathrm{~cm}^{-3} \mathrm{~s}^{3}\end{array}$ \\
\hline 5.5 & 1.72 & 5.0 & 5236 & 2.391 & 0.716 & 0.832 & 2.87 & $1.226 \mathrm{e} 6$ \\
6 & 1.72 & 3.85 & 5530 & 2.405 & 0.752 & 0.867 & 2.77 & $1.219 \mathrm{e} 6$ \\
7 & 1.85 & 3.10 & 5181 & 2.396 & 0.707 & 1.09 & 2.20 & $1.226 \mathrm{e} 6$ \\
8 & 1.95 & 3.05 & 5132 & 2.403 & 0.699 & 1.35 & 1.78 & $1.225 \mathrm{e} 6$ \\
9 & 2 & 3 & 5095 & 2.398 & 0.695 & 1.58 & 1.52 & $1.219 \mathrm{e} 6$ \\
10 & 2.04 & 2.99 & 5084 & 2.401 & 0.693 & 1.79 & 1.34 & $1.218 \mathrm{e} 6$ \\
11 & 2.07 & 2.98 & 5075 & 2.402 & 0.691 & 1.96 & 1.23 & $1.226 \mathrm{e} 6$ \\
12 & 2.08 & 2.97 & 5065 & 2.402 & 0.690 & 2.14 & 1.12 & $1.218 \mathrm{e} 6$ \\
\hline
\end{tabular}

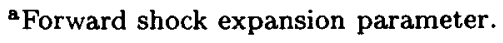

${ }^{b}$ Ejecta core density in velocity space. 
Table 5. Models $r_{b}=2.35 \mathrm{pc}, \rho_{\text {core }}=2.25 e 6 \mathrm{~g} \mathrm{~cm}^{-3} \mathrm{~s}^{3}$, and $\rho r_{b}^{2}=14 \mathrm{H}_{\text {atom cm}} \mathrm{cm}^{-3} \mathrm{pc}^{2}$

\begin{tabular}{ccccccccc}
\hline \hline$n$ & $\begin{array}{c}M_{e j} \\
M_{\odot}\end{array}$ & $\begin{array}{c}E_{51} \\
10^{51} \mathrm{ergs}\end{array}$ & $\begin{array}{c}v_{b}(320 \mathrm{yrs}) \\
\mathrm{km} \mathrm{s}^{-1}\end{array}$ & $\begin{array}{c}r_{b}(320 \mathrm{yrs}) \\
\mathrm{pc}\end{array}$ & $\eta^{\mathrm{a}}$ & $\begin{array}{c}r_{r}(320 \mathrm{yrs}) \\
\mathrm{pc}\end{array}$ & $r_{b} / r_{r}$ & $\begin{array}{c}M_{e j}(n-3) / n / v_{\text {core }}^{3} \mathrm{~b} \\
\mathrm{~g} \mathrm{~cm}^{-3} \mathrm{~s}^{3}\end{array}$ \\
\hline 5.5 & 1.815 & 4.15 & 5134 & 2.345 & 0.716 & 1.28 & 1.92 & $2.146 \mathrm{e} 6$ \\
6 & 1.815 & 2.8 & 5395 & 2.347 & 0.752 & 1.21 & 1.94 & $2.248 \mathrm{e} 6$ \\
7 & 1.875 & 2.15 & 5338 & 2.349 & 0.744 & 1.29 & 1.82 & $2.256 \mathrm{e} 6$ \\
8 & 1.95 & 2.05 & 5212 & 2.349 & 0.726 & 1.44 & 1.63 & $2.223 \mathrm{e} 6$ \\
9 & 2 & 2 & 5153 & 2.345 & 0.719 & 1.59 & 1.48 & $2.240 \mathrm{e} 6$ \\
10 & 2.035 & 1.985 & 5130 & 2.348 & 0.715 & 1.73 & 1.36 & $2.238 \mathrm{e} 6$ \\
11 & 2.065 & 1.965 & 5105 & 2.347 & 0.712 & 1.85 & 1.27 & $2.267 \mathrm{e} 6$ \\
12 & 2.075 & 1.95 & 5085 & 2.347 & 0.709 & 1.96 & 1.20 & $2.267 \mathrm{e} 6$ \\
\hline
\end{tabular}

a Forward shock expansion parameter.

${ }^{b}$ Ejecta core density in velocity space. 